\title{
Tracking the Early Stages of Massive Star Formation
}

\author{
Serena Viti \\ Department of Physics and Astronomy, University College London, Gower Street, \\ WC1E 6BT, UK \\ email: sv@star.ucl.ac.uk
}

\begin{abstract}
Until recently hot cores were the first indirect manifestation of a young massive star, but recent observational successes are now providing us with the first candidates for the very early phases of massive star formation, probably the precursors of hot cores. The characteristic hot core chemistry is believed to arise from the evaporation of the icy mantles that accumulate on the dust grains during the collapse the leads to the formation of the massive star. The duration over which the grains are warmed is determined by the time taken for a pre-stellar core to evolve to the Main Sequence. Hence, hot cores and their precursors contain an integrated record of the physics and chemistry occurring during the collapse that led to the star. In this article I will review recent advances in the chemical modelling of hot cores and their precursors in light of the recent TPD experiments on a variety of ices and I will briefly discuss the implications of such studies for the interpretation of high mass protostellar objects.
\end{abstract}

Keywords. astrochemistry — ISM: clouds — ISM: molecules — stars: formation — stars: pre-main sequence

\section{Introduction}

Unlike the case of low-mass stars, the process that leads to the formation of a massive star is not well understood. This is partly due to the fact that observationally it is still rather difficult to classify the early stages of massive star formation as we routinely do for low-mass stars; e.g., there is not yet a definite equivalent of a class 0 or a class I for massive stars, although recent observational successes may be providing us the first candidates for such classification (e.g., Garay et al. 2004; Faundez et al. 2004; Sridharan et al. 2005; see also chapter by Kurtz in this volume). The process that leads to the formation of a high mass star, whether it is by accretion (free-fall or accelerated collapse, e.g., Lintott et al. 2005), or coalescence and accretion (Bonnell et al. 1998), must be extremely fast, $\sim 10^{4}-10^{5} \mathrm{yr}$, and should depend on the final mass of the star (e.g., Bernasconi \& Maeder, $1996)$. Thus, the contraction of a core from a low to a very high density $\left(\sim 10^{7} \mathrm{~cm}^{-3}\right)$ occurs very quickly and the hydrogen starts burning while the new born star is still well embedded in the parent cloud, which is probably still collapsing. The star will reach ZAMS (Zero Age Main Sequence, which is when the star reaches its minimum radius, its maximum mass and its hottest effective temperature) while still embedded and the dust surrounding the star is still very abundant. Hence a star may be quite evolved but have still enough dust to have the same spectral energy distribution of a younger object, making the task of detecting the early stages of high-mass star formation a hard one. In addition, it is well known that massive stars do not form in isolation but rather in so called OB associations, large aggregates of hundreds to several thousand young stars.

Nevertheless, there are indeed indirect ways of tracking and studying the early stages of a massive star, when still embedded. Until a couple of decades ago, massive star formation 
was traced by the detection of compact and ultracompact $\mathrm{H}$ II regions (UCHII), regions of dense gas ionized by the newly formed star. However, UCHII only trace the late stages of the formation of a massive star. Some two decades ago molecular observations in the submillimeter revealed the presence of the so called 'hot cores', remnants of the collapsing cloud in the process of formation of the massive star. These cores, in principle, may enable us to sample the pre-stellar material and infer the conditions in the natal cloud prior to the collapse. Hot cores are small $\left(\sim 10^{-2}-10^{-1} \mathrm{pc}\right)$, dense $\left(\geqslant 10^{7} \mathrm{H}_{2} \mathrm{~cm}^{-3}\right)$, relatively warm $\left(\geqslant 10^{2} \mathrm{~K}\right)$, optically thick $\left(A_{V} \geqslant 10^{2} \mathrm{mag}\right.$. $)$, and transient $\left(\leqslant 10^{5} \mathrm{yr}\right)$ cores. They show a richer chemistry than that found in quiescent lower density $\left(\sim 10^{3}-10^{5} \mathrm{~cm}^{-3}\right)$ and cool $(\sim 10 \mathrm{~K})$ molecular clouds. For example, in hot cores the abundance of small saturated molecules (e.g., $\left.\mathrm{H}_{2} \mathrm{O}, \mathrm{NH}_{3}, \mathrm{H}_{2} \mathrm{~S}, \mathrm{CH}_{3} \mathrm{OH}\right)$ and large organic species $\left(\mathrm{CH}_{3} \mathrm{CN}\right.$, $\left.\mathrm{CH}_{2} \mathrm{CHCN}, \mathrm{CH}_{3} \mathrm{CH}_{2} \mathrm{CN}, \mathrm{C}_{2} \mathrm{H}_{5} \mathrm{OH}\right)$ can be enhanced by over two orders of magnitude. Such high abundances are believed to arise from the evaporation, induced by the nearby (proto)star, of ice mantles frozen-out on to the dust grains during the collapse of the parent cloud. Since the freeze-out timescale is short compared to the chemical timescale, material freezing-out includes reactive species that may undergo further processing on the ice, in particular hydrogenation (although at high densities oxidation may also occur). So, for example, atomic $\mathrm{N}$ can be converted to $\mathrm{NH}_{3}$, oxygen to water and so on. Large organics may then arise from a more drastic processing of the molecular ice. In summary, there are three main processes that affect and characterize the chemistry of hot molecular cores: depletion (or freeze-out on to grains); surface reactions; and thermal evaporation. A detailed interpretation of the observed molecular abundances require chemical models that take into account all three of these processes.

Since their discovery, hot cores have been studied intensively, both observationally and theoretically (see e.g., reviews by Walmsley \& Schilke 1993; Millar 1993; Kurtz et al. 2000). Models of hot cores in which a collapse phase including chemistry and freeze-out is followed by a static warm phase in which evaporation and gas-phase chemistry occurs have had considerable success in understanding their chemical richness (see for example Brown et al. 1988; Caselli et al. 1993; Charnley 1997; Hatchell et al. 1998). Although these studies do differ in the emphasis given to the chemistry in pre- and post-stellar phases, a common assumption has been that the evaporation of the ices occurs effectively instantaneously. The justification for such assumption goes as follow: if the evaporation is driven by the stellar radiation field, then the grain temperature must rise rapidly from around $10 \mathrm{~K}$ (in the molecular cloud) to around $200 \mathrm{~K}$ (in the hot core). However, such assumption may not be always appropriate as I will show in the next two sections.

\section{Thermal Desorption of Ices}

The instantaneous thermal evaporation approximation has always been adopted because contraction of a massive core occurs so quickly that hydrogen burning starts while the new-born star is still well embedded in the parent cloud which is probably still collapsing (Hanson, 1998). However, the contraction time $\left(t_{c}\right)$, i.e the time after which hydrogen starts burning and the star becomes a ZAMS, is not well known for hot stars and certainly its dependence on the initial mass of the star has not yet been defined. In fact, it is unlikely to be less than 20,000 years even for the most massive stars. In such a time different species will then evaporate at different times.

Viti \& Williams (1999) explored the idea that the duration in which the grains are warmed from very low $(\sim 10 \mathrm{~K})$ temperatures to the temperature at which $\mathrm{H}_{2} \mathrm{O}$ ice desorbs and above, is determined by the time taken for a pre-stellar core to evolve towards the Main Sequence and found that the chemistry of the hot cores must reflect 
this temperature-driven evolution; the evolutionary chemistry can therefore provide, in principle, a direct measure of the stellar turn-on time. Thus, the formation of hot cores contains a record of the collapse process as well as the ignition history of the star. Their models, in the absence of reliable data, made a gross simplification in the description of desorption from mixed ices; however, from their study it was already clear that the first $\sim 60,000$ years of star formation differ significantly among models with different contraction times and that some key species show strong selective effects, in particular ions, sulfur-bearing species and some large molecules such as $\mathrm{CH}_{3} \mathrm{OH}$ and $\mathrm{CH}_{3} \mathrm{CN}$.

Moreover, we know that shocks in the form of molecular outflows appear at an early stage of high-mass protostellar evolution (e.g., Cesaroni et al. 1999). Hence it is possible that a slow shock could propagate through a hot core before or during its warming up phase, removing the grain mantles. Since the order of onset of hydrogen burning, outflows, and reaching the ZAMS in high-mass star formation is unknown, a core could be shocked before or during the thermal heating stage. Viti et al. (2001) have investigated whether it is possible to infer if and when a shock passed through a hot core by the use of chemical models. They find that, as in previous work (e.g., Hatchell et al. 1998), the fractional abundances of various sulfur-bearing species is quite sensitive to the environmental influences. This was followed up by Hatchell \& Viti (2002) who looked for variations in the NS/CS ratio in a sample of hot core sources. However, although the NS/CS value found excluded the 'standard' scenario (where everything evaporates at once) and indicated that hot cores are shocked at an early stage and that they are short-lived, this ratio was the same throughout the sample. This was a consequence of the fact that their sample was biased by their common association with UCHII regions, usually indicating an already evolved stage of high-mass star formation. What the Hatchell \& Viti (2002) study clearly indicated, however, is that the selective chemical effects that should occur during the temperature increase of the dust grains will be relevant during the formation of a hot core rather than at a late stage when the hot core is already formed. In simple terms we can view the hot cores as the third stage of massive star formation with massive cold cores and high-mass protostellar objects (HMPOs) as the first two phases (but see chapter by Kurtz in this volume for a more thorough description of the different phases).

\section{Chemical Characterization of HMPOs}

From the mid-1990s HMPOs have been routinely discovered in systematic surveys (Molinari et al. 2002 and references therein; Sridharan et al. 2002; Beuther et al. 2002). Whether or not all HMPOs are in fact hot cores precursors, it is now clear that HMPOs and hot cores contain the history of the early phases of high-mass star formation. It is also probable that HMPOs are cooler than hot cores, hence that modelling such phases must take into consideration the time dependent increase of the temperature. Then, the time variation in the abundance ratio of evaporated molecular species can be used as a 'chemical clock' which may constrain the contraction time of hot stars.

The initial studies on time dependent evaporation already emphasized that appropriate temperature programmed desorption (TPD) experiments on mixed ices under near-interstellar conditions are essential. The recent observational searches for cold and warm massive cores highlight such needs even more. TPD measurements on a variety of ices have now been made (Fraser et al. 2001; Collings et al. 2003; Collings et al. 2004), and showed that the assumptions made in the models are indeed wrong (see Collings \& McCoustra in this volume). In addition, the properties of some species for which experiments are not yet available have been considered (Collings et al. 2004). In summary, it was found that it is possible to categorize all species and that, depending on what 

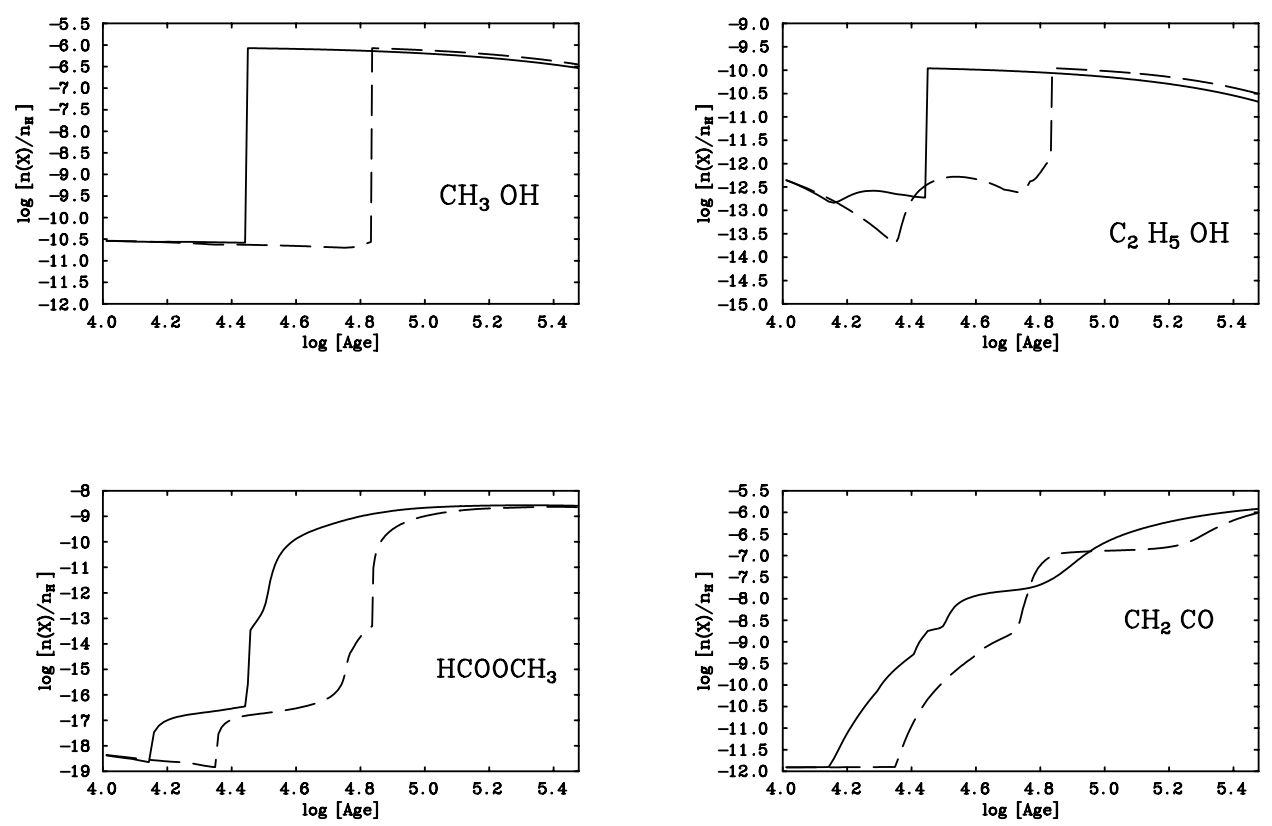

Figure 1. Fractional abundances of selected large molecules as a function of time for a $25 \mathrm{M}_{\odot}$ (solid line) and a $10 \mathrm{M}_{\odot}$ (dotted line) star.

category a species belongs to, desorption occurs in as many as four temperature bands. The proportion of each species that evaporates at each band depends on the total amount present on the grains at the time the temperature starts increasing from $10 \mathrm{~K}$. These recent experimental results have now been incorporated into chemical models of high-mass star formation (Viti et al. 2004) and it was found that distinct chemical events occur at specific grain temperatures and these differ depending on the mass of the star. The details of such models can be found in Viti et al. (2004), but here it is worth noting some of the differences that appropriate TPD measurements can make to the abundances of some species. Figure 1 shows the time evolution of the fractional abundance of four molecular species for a $25 \mathrm{M}_{\odot}$ (solid line) and a $10 \mathrm{M}_{\odot}$ (dotted line). From the figure we note that large species are in fact good indicators of old cores as these strongly bound species are abundant only in the gas at late times. This implies that in HMPOs we should not find the same chemical richness of organic species that is routinely found in hot cores. However, the species that are most affected by the inclusion of the new evaporation treatment are, not surprisingly, sulfur-bearing species. In fact while larger species such as methanol and $\mathrm{CH}_{2} \mathrm{CO}$ maintain the same trends from a $5 \mathrm{M}_{\odot}$ to a $25 \mathrm{M}_{\odot}$ star, the behavior of sulfur bearing species strongly depend on the heating rate and hence on the mass of the star. This can be of great relevance to the problem of sulfur depletion, as we shall see in the next section.

\subsection{The Sulfur Problem}

It is often assumed that simplest species, once frozen out, react with hydrogen atoms to form saturated compounds. However, recently, doubts have been raised regarding the 

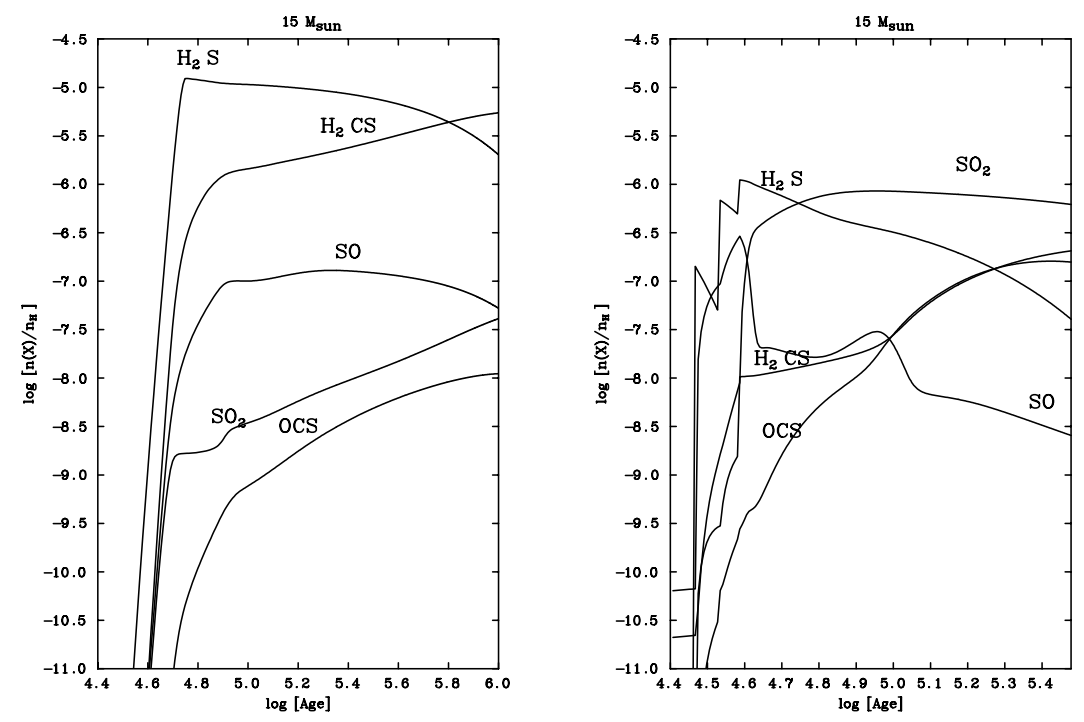

Figure 2. Time evolution of selected sulfur bearing species for the Vitti et al. (2004) models (right panel) and for the Vitti \& Williams (1999) models (left panel).

form that sulfur takes once it depletes onto the grains. Attempts at detecting $\mathrm{H}_{2} \mathrm{~S}$ on ices with the Infrared Space Observatory have been unsuccessful (van Dishoeck \& Blake 1998). In fact, the only sulfur bearing species detected in the solid state has been OCS (Palumbo et al. 1997), with an abundance of $\sim 10^{-7}$. The possibility that OCS could be the main reservoir of sulfur on the grains seems to have gained support recently by observations of the envelopes of massive young stars (van der Tak et al. 2003). Other species have been proposed as the main reservoir of sulfur on grains. For example, iron sulfide (FeS) grains have recently been discovered in protoplanetary disks and proposed as the reservoir of sulfur (Keller et al. 2002). However, there is no doubt that $\mathrm{H}_{2} \mathrm{~S}$ is abundant in hot cores (e.g., Hatchell et al. 1998) and pure gas-phase models fail to reproduce it in the abundance observed; hence there seems to be little alternative but for some sulfur to hydrogenate and form $\mathrm{H}_{2} \mathrm{~S}$ once depleted. In fact, according to the Viti et al. (2004) models, by taking into consideration the selective chemical nature of time dependent evaporation, sulfur bearing species vary by substantial amount during the evolution of massive stars. Figure 2 shows the time evolution of selected sulfur bearing species for the Viti et al. (2004) models (right panel) and for the Viti \& Williams (1999) models (left panel). Both plots show that sulfur bearing species vary in abundances with time as a consequence of the time dependent nature of the mantle evaporation but, the Viti et al. (2004) model, which incorporates the latest TPD experiments from Collings et al. (2004), shows much more variation in the gas-phase abundances of sulfur-bearing species. Although, to start with, all the main source of sulfur on the grains is $\mathrm{H}_{2} \mathrm{~S}$, its evaporation at different evolutionary stages affects all other sulfur bearing species: In the $25 \mathrm{M}_{\odot}$ star model, for example, the abundance of $\mathrm{H}_{2} \mathrm{~S}$ itself varies by about one order of magnitude with time (see Figure 1 in Viti et al. 2004). This difference becomes larger as the mass of the star decreases, as can be seen from Figure 2. This fast and changing behavior has several consequences on other sulfur bearing species including OCS, which becomes abundant, even more than $\mathrm{H}_{2} \mathrm{~S}$, for older cores, indicating that the 


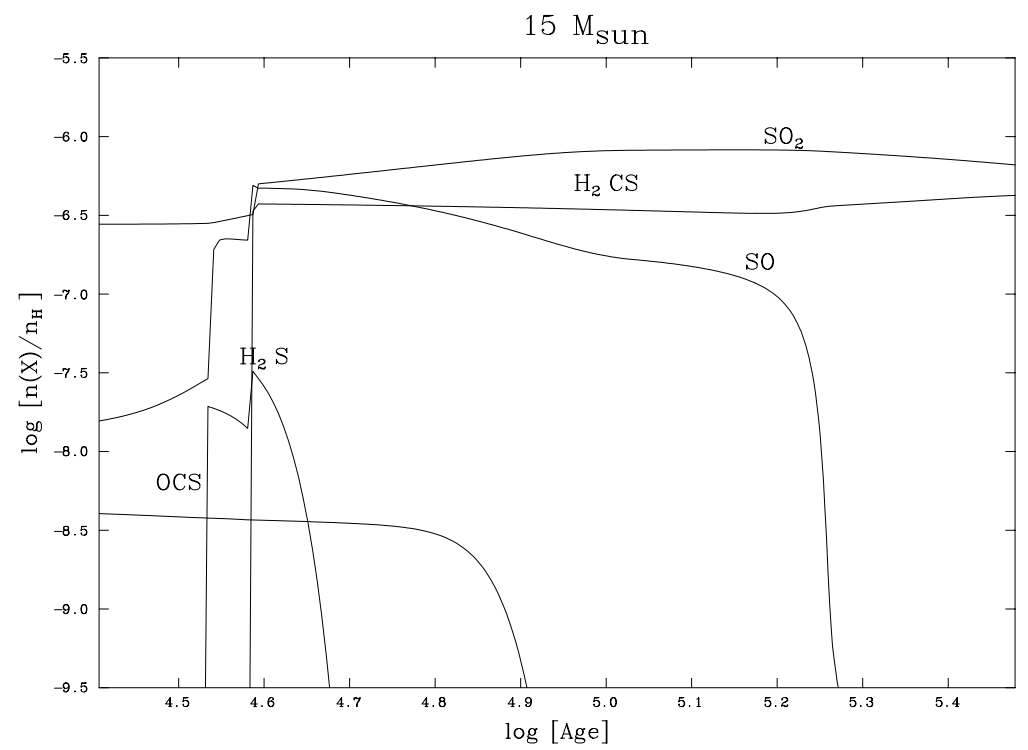

Figure 3. Time evolution of selected sulfur bearing species for a model where surface reactions during the collapse as well as the warm up phase are included (Viti, Garrod, \& Herbst, in preparation).

high abundances of OCS observed by, for example, van der Tak (2003) may not lead necessarily to the conclusion that OCS is evaporated directly from the icy mantles.

The highly-reactive nature of sulfur bearing species during the HMPOs evolution means that although it is true that ratio of sulfur bearing species can be used as a time 'tracer', one must pay particular attention to the chemical treatment of desorption. In fact, it is possible that, for some species, the simple assumption of hydrogenation as the only type of surface reaction occurring during the formation of a hot core is not valid. Chemical models where more complex surface reactions are included have been presented before: Caselli et al. (1993), for example, follow the chemistry which consists of both gasphase and grain-surface processes during the isothermal collapse that leads to the star, and purely gas-phase processes after the surrounding region becomes hot. They show that a better understanding of grain chemical processes is necessary in order to account even for the most abundant simple species. In fact, it is also likely that surface reactions continue to take place during the initial increase of the temperature, at the same time as thermal desorption. This is not usually taken into account in chemical models of massive star formation, but it turns out that it may be important, especially for sulfur-bearing species. Figure 3 shows an example of a new preliminary model where more complex surface reactions were also included during the warm up phase. The models used for Figures 2 and 3 only differ in their treatment of surface reactions: the models in Figure 2 only include surface reactions during the collapse phase and they consist only of fast hydrogenation (e.g., any oxygen atom that sticks to the surface hydrogenate to water), while, for the model of Figure 3, a more thorough treatment of surface reactions, using the Ohio surface reaction rate file (Ruffle et al. 2000) was used and surface reactions continue into the warm up phase. A comparison of the two figures clearly shows that there is still plenty to learn about the role of surface chemistry! 
The few examples given above clearly indicate that the uncertainties on the initial conditions during collapse such as the freeze out efficiency, cosmic ray induced desorption, as well as surface reactions make the problem harder: ratios of selected species as 'chemical clocks' can therefore be useful only if one first can calibrate such clocks, as also shown by Wakelam et al. (2004). In summary, the recent advances discussed above clearly indicate that chemical differentiation can be used as evolutionary tracer only if (i) a large sample of massive cores at different evolutionary ages is available and (ii) several molecular species are used at once. In the next section I will briefly mention one example of work in progress that aims at calibrating chemical clocks.

\subsection{The $\mathrm{CH}_{3} \mathrm{OH} / \mathrm{CH}_{3} \mathrm{CN}$ Ratio in a Sample of HMPOs}

Theoretical studies (Viti \& Williams 1999; Viti et al. 2004) showed that the time variation in the abundance ratio of selected evaporated molecular species could provide us with a 'chemical clock' which could constrain the contraction time of hot stars. In particular, $\mathrm{CH}_{3} \mathrm{OH} / \mathrm{CH}_{3} \mathrm{CN}$ and NS/CS were found to be particularly sensitive to the pre-hot-core phase and to whether a shock chemistry occurs, respectively. An extended observational survey of $\mathrm{CH}_{3} \mathrm{OH} / \mathrm{CH}_{3} \mathrm{CN}$ in a sample of HMPOs has now been carried out (Beardsmore et al. in preparation). The aim of this survey was quite simple: measure and compare the $\mathrm{CH}_{3} \mathrm{OH} / \mathrm{CH}_{3} \mathrm{CN}$ ratio in a sample of probable HMPOs believed to be at different evolutionary stages. These two species were chosen because their detection would immediately confirm their identity as high-density warm cores, as both species are not easily produced in the gas-phase chemistry of dark clouds. Moreover, TPD experiments have shown that $\mathrm{CH}_{3} \mathrm{CN}$ comes off the dust grains earlier than $\mathrm{CH}_{3} \mathrm{OH}$ and can therefore be observed individually during that time. Observations of G29.96-0.02 (Pratap et al. 1999) and G9.62+ 0.19 (Hofner et al. 2001) show that the $\mathrm{CH}_{3} \mathrm{CN}$ in the hot core is displaced from the $\mathrm{CH}_{3} \mathrm{OH}$ peak which in both cases appears to arise from an unrelated source; this could be due to time-dependent evaporation. The sample of Beardsmore et al. was selected from a larger sample of 73 northern methanol masers (Walsh et al. 2003) and included 9 UCHII-associated and 9 unassociated cores, all with similar high mass and luminosity and dust temperature in the range 30-300K, i.e. sufficiently hot for the selective desorption of grain-mantle molecules to be occurring. Two molecular clumps from the list of Molinari et al. (1996), the so-called 'high' sources, believed to be at an even younger evolutionary stage than the cores not associated with UCHII, were also selected.

Preliminary results indeed show that the $\mathrm{CH}_{3} \mathrm{OH} / \mathrm{CH}_{3} \mathrm{CN}$ ratio varies substantially among objects (see Figure 4) and that the cooler (youngest?) objects (such as IRAS 18089-1732 and IRAS 18236-1205) also have the lowest $\mathrm{CH}_{3} \mathrm{OH} / \mathrm{CH}_{3} \mathrm{CN}$ ratio. However, it is worth noting that we are still uncertain about the routes of formation of $\mathrm{CH}_{3} \mathrm{CN}$, which probably occurs on grains. Hence models tend to underestimate its abundance so, although at a first sight, this result may be consistent with a young age when methanol has yet to evaporate completely, our inability to account for the $\mathrm{CH}_{3} \mathrm{CN}$ abundance observed in evolved hot cores makes this ratio an unreliable calibrator on its own. Nevertheless Figure 4 indicates that, unlike with the Hatchell \& Viti (2002) study, the ratio of $\mathrm{CH}_{3} \mathrm{OH} / \mathrm{CH}_{3} \mathrm{CN}$ does vary by over one order of magnitudes among the selected objects.

Similar efforts have been carried out by Araya et al. (2005) who conducted a survey in $\mathrm{CH}_{3} \mathrm{CN}$ for hot and dense molecular cores towards a sample of 17 southern sources with the aim of identifying very young sites of massive star formation. They use $\mathrm{CH}_{3} \mathrm{CN}$ as an tracer of warm massive cores and they find that at least 4 of their sources are new candidates for hot molecular cores (although probably at a quite evolved stage). 


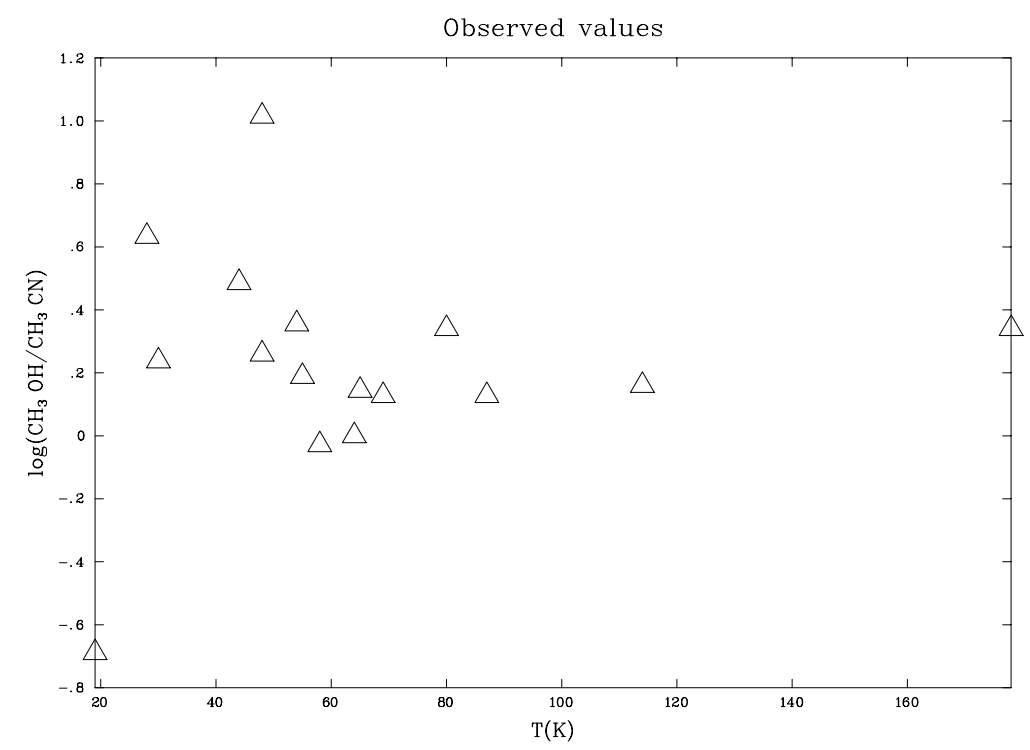

Figure 4. $\mathrm{Log}$ ratio of fractional abundances of methanol and $\mathrm{CH}_{3} \mathrm{CN}$ for a sample of HMPOs as a function of their estimated temperature.

\section{Conclusions}

Over the last few years, studies of high-mass star formation have advanced enormously, and we are now at the stage where the cold sites of massive star formation may have been detected (see chapter by Kurtz in this volume). At the same time chemical models have evolved to a stage where surface reactions and thermal desorption need to be properly treated and help from experiments must be sought. The complexity of massive star formation is such that in order to use chemical models as an evolutionary tool to track high-mass protostellar objects they must include a proper treatment of evaporation of the icy mantle. The icy mantle also needs to be better characterized as the initial conditions (initial density, collapse, depletion, etc.) significantly impact its composition during and at the end of the collapse phase. These uncertainties are of particular importance for S-bearing species, which are often proposed as evolutionary tracers or 'chemical clocks'.

\section{Acknowledgements}

SV would like to thank PPARC for the financial support of an individual advanced fellowship.

\section{References}

Araya, E., Hofner, P., Kurtz, S., Bronfman, L., \& DeDeo, S. 2005, Ap. J. Suppl. 157, 279 Bernasconi, P.A. \& Maeder, A. 1996, A\& A 307, 829

Beuther, H., Schilke, P., Menten, K.M., Motte, F., Sridharan, T.K., \& Wyrowski, F. 2002, Ap. J. 566,945

Bonnell, I., Bate, M.R., \& Zinnecker, H. 1998, MNRAS 298, 93

Brown, P.D., Charnley, S.B., \& Millar, T.J. 1988, MNRAS 231, 409

Caselli, P., Hasegawa, T.I., \& Herbst, E. 1993, Ap. J. 408, 548

Cesaroni, R., Felli, M., Jenness, T., et al. 1999, A $\dot{E A}$ 345, 949

Charnley, S.B. 1997, Ap. J. 481, 396

Collings, M.P., Dever, J.W., Fraser, H.J., \& McCoustra, M.R.S. 2003, Ap. J. 583, 1058 
Collings, M.P., Anderson, M.A., Chen, R., Dever, J.W., Viti, S., Williams, D.A., \& McCoustra, M.R.S. 2004, MNRAS 354, 1133

Faundez, S., Bronfman, L., Garay, G., Chini, R., Nyman, L.-A., \& May, J. 2004, A $8 A$ 426, 97

Fraser, H.J., Collings, M.P., McCoustra, M.R.S., \& Williams, D.A. 2001, MNRAS 327, 116

Garay, G., Faundez, S., Mardones, D., Bronfman, L., Chini, R., \& Nyman, L.-A. 2004, Ap. J. 610,313

Hanson, M.M. 1998, Bolder-Munich II: Properties of Hot, Luminous Stars ed. I. Howarth (ASP Conf. Ser. 131), 1

Hatchell, J. Thompson, M.A., Millar, T.J., \& Mcdonald, G.H. 1998, A\&A 338, 713

Hatchell, J. \& Viti, S. 2002, A $\mathscr{E} A$ 381, L33

Hofner, P., Wiesemeyer, H., \& Henning, T. 2001, Ap. J. 549, 425

Keller, L.P., Hony, S., Bradley, J.P., Molster, F.J., Waters, L.B.F.M. et al. 2002, Nature 417, 148

Kurtz, S., Cesaroni, R., Churchwell, E., Hofner, P., \& Walmsley, C.M. 2000, in Protostars and Planets IV, eds. V. Mannings, A.P. Boss, \& S.S. Russell, p. 299

Lintott, C.J., Viti, S., Rawlings, J.M.C., Williams, D.A., Hartquist, T.W., Caselli, P., Zinchenko, I., \& Myers, P. 2005 Ap. J. 620, 795

Millar, T.J. 1993, in Dust and Chemistry in Astronomy, eds. T. Millar \& D. Williams (Istitute of Physics, Bristol), p. 249

Molinari, S., Brand, J., Cesaroni, R., \& Palla, F. 1996, A\&A 308, 573

Molinari, S., Testi, L., Rodriguez, L.F., \& Zahang, Q. 2002, Ap. J. 570, 758

Palumbo, M.E., Geballe, T.R., \& Tielens, A.G.G.M. 1997, Ap. J. 479, 839

Pratap, P., Megeath, S.T., \& Bergin, E.A. 1999, Ap. J. 517, 799

Ruffle, D.P. \& Herbst, E. 2000, MNRAS 319, 837

Sridharan, T. K., Beuther, H., Schilke, P., Menten, K.M. \& Wyrowski, F. 2002, Ap. J. 566, 931

Sridharan, T.K., Beuther, H., Saito, M., Wyrowski, F., \& Schilke, P. 2005, Ap. J., submitted, astro-ph/0508421

van der Tak, F., Boonman, A.M.S., Braakman, R., \& van Dishoeck, E.F. 2003, A\&\&A 412, 133

van Dishoeck, E. \& Blake, J. 1998, ARAESA 36, 317

Viti, S. \& Williams, D.A. 1999, MNRAS, 305, 755

Viti, S., Caselli, P., Hartquist, T.H., \& Williams, D.A. 2001, A $\& A$ 370, 1017

Viti, S., Collings, M.P., Dever, J.W., McCoustra, M.R.S., \& Williams, D.A. 2004, MNRAS 354, 1141

Wakelam, V., Caselli, P., Ceccarelli, C., Herbst, E., \& Castets, A. 2004 A $\& A$ A 422, 159

Walmsley, C.M. \& Schilke, P. 1993, in Dust and Chemistry in Astronomy, eds. T. Millar and D. Williams (Istitute of Physics, Bristol), p. 37

Walsh, A.J., Macdonald, G.H., Alvey, N.D.S., Burton, M.G., \& Lee, J.-K. 2003, A\&A A 410, 597

\section{Discussion}

GEBALLE: You did not explicitly mention photolysis, was it included in your modelling of grain warm-up? Is it also important in grain chemistry?

VITI: No, photolysis is not included in these models. Although it may indeed be important, I think that in the case of hot cores and their precursors thermal desorption will dominate. 


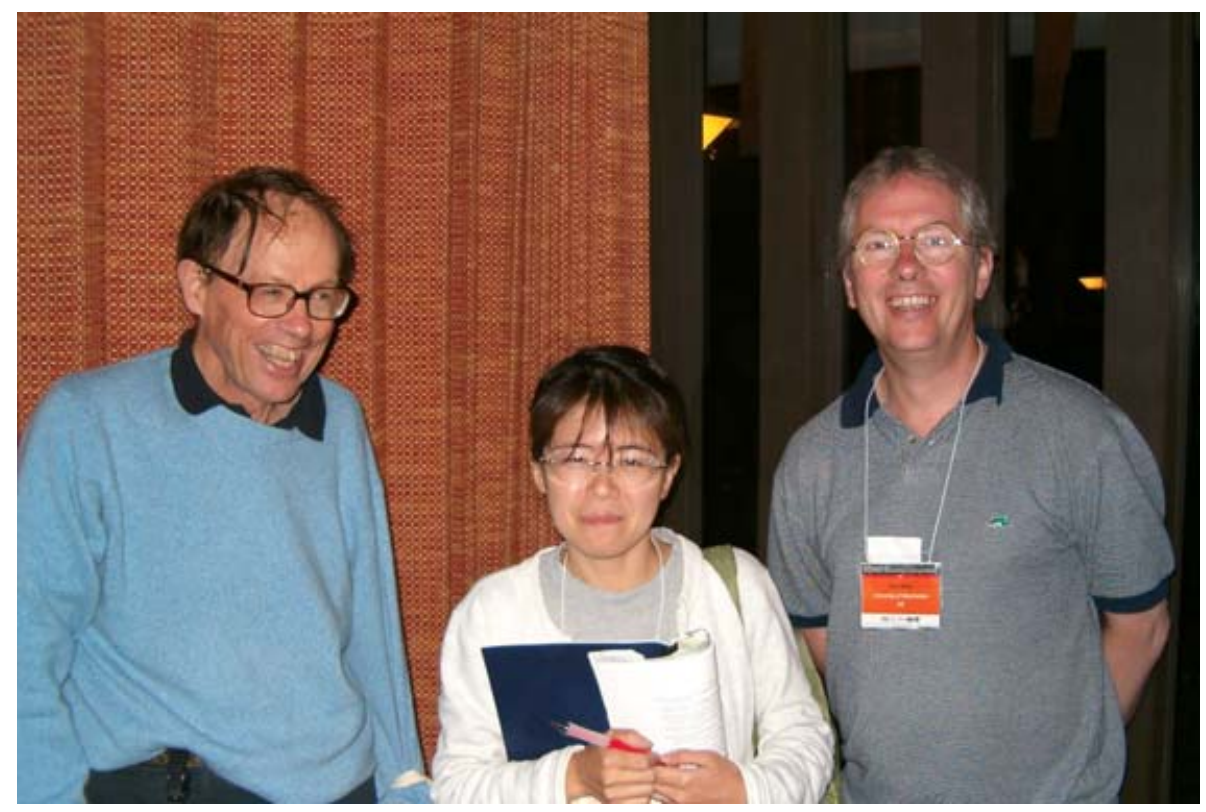

Photo: E. Herbst 